\title{
Isolated ovarian actinomycosis - a diagnosis often underlooked
}

\section{Vijaya C, Shetty A, Geethamani V}

\begin{abstract}
Pelvic Actinomycosis is rare, and isolated ovarian actinomycosis even rarer. We present an interesting case of a 39 year old lady, who was extensively worked up, following a clinical suspicion of ovarian cancer. The postoperative diagnosis was ovarian actinomycosis. In majority of cases, including ours, the indolent clinical course, together with the malignant tumour like appearance at imaging was misleading, and a final diagnosis of actinomycosis was made only after the histological examination of the specimen. Although ovarian actinomycosis is often related to the use of intra-uterine device (IUD), the index case did not have any history of using IUD, also there was no evidence of actimomycosis elsewhere, including abdomen. We present this case, not only for its diagnostic dilemma and rarity, but also to highlight the fact that a high suspicion for actinomycotic infection can spare the patient from radical and excessive surgical intervention.
\end{abstract}

Keywords: ovarian actinomycosis, intra-uterine device, malignancy, histopathology

\section{Introduction}

Actinomycosis is a suppurative, chronic granulomatous disease, caused by any of several anaerobic organisms from the genus Actinomyces. Pelvic actinomycosis is usually insidious and is often mistaken for pelvic abscess or ovarian malignant tumours ${ }^{1}$. Use of intrauterine device (IUD) is considered a risk factor in ovarian actinomycosis. We present a case of a 39 year old lady, in the absence of history of IUD usage, presenting with abdominal pain, which was worked up keeping in mind a diagnosis of ovarian malignancy.

\section{Case report}

A 39 year old lady presented to the obstetric clinic with lower abdominal

Department of Pathology, Sapthagiri Institute of Medical Sciences and Research Center, Bangalore, India.

Correspondence: Archana Shetty

E-mail: archanashetty@yahoo.com

Competing interests: None pain and painful micturition for one week duration. She was not married and did not give any history of using an IUD. She had history of fitting of artificial incisor teeth 4 years ago. In view of possible underlying pelvic tuberculosis or malignancy, the patient was investigated with the following findings. Blood: Mild leucocytosis and thrombocytosis. Ultrasonography (USG) abdomen and pelvis: Right sided adnexal mass with internal echoes, measuring $7.5 \mathrm{~cm} \times 4 \mathrm{~cm}$, suspicious of inflammatory pathology. CT (Computed Tomography) Abdomen: Well defined solid enhancing mass lesion, involving the right ovary, measuring $7 \times 5 \times 3 \mathrm{~cm}^{3}$, with cystic components. No fat stranding seen. CA-125 was done and was normal.

A provisional diagnosis of ovarian tumour/ PID was made and the patient was planned for a diagnostic laparoscopy. On the table, a right tubo-ovarian mass was seen with dense adhesions to the surrounding structures, including the sigmoid colon. Hysterectomy with right sided salphingoophorectomy was done along with adhesiolysis. Left adnexal was unremarkable.

\section{Figure 1. CT abdomen and pelvis showing the left adnexal mass with an attached cyst (arrow)}

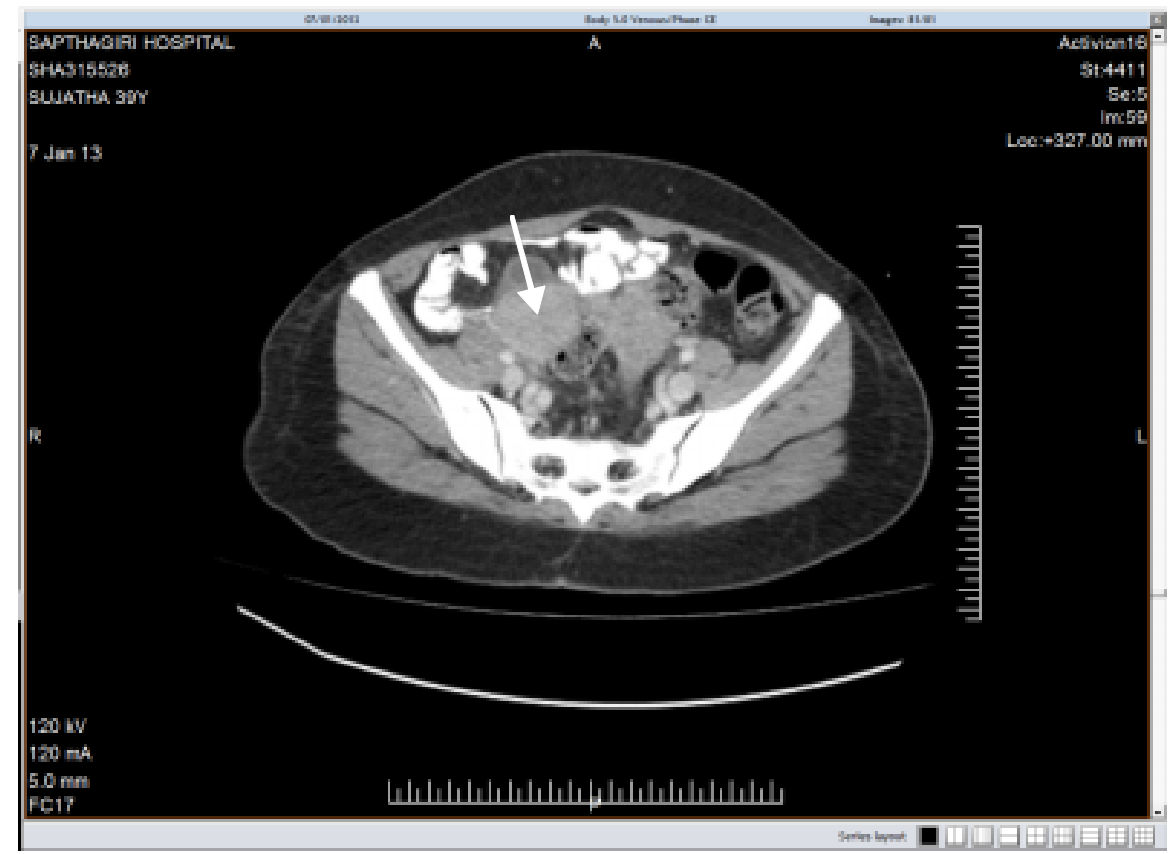


Figure 2. Cut section of the adnexal mass showing yellowish solid areas with an attached haemorrhagic cyst

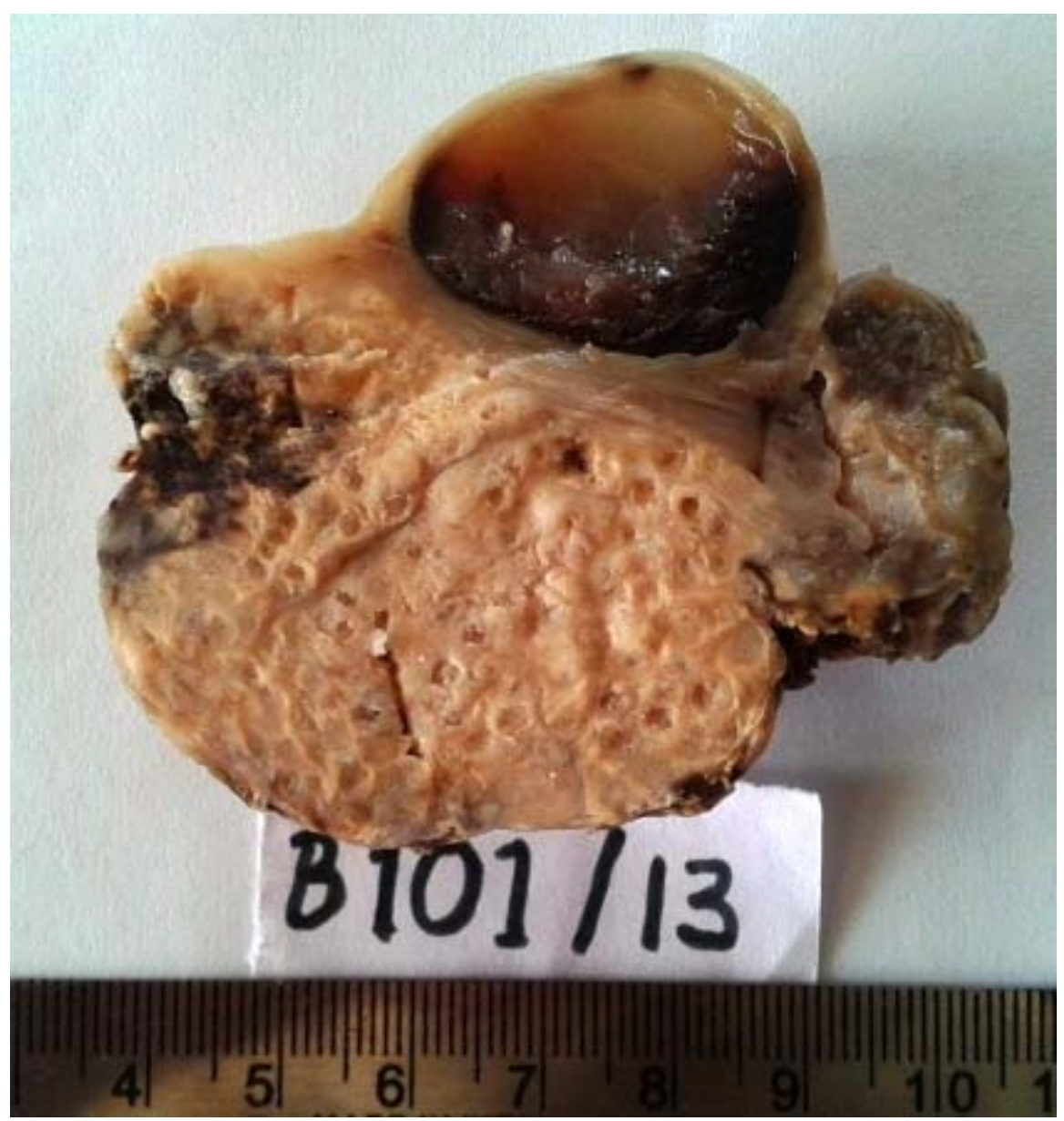

Morphological examination showed the uterus with cervix measuring $7 \times$ $3 \times 2 \mathrm{~cm}^{3}$ with normal endometrial cavity and cervix. Right adnexal mass measured $7 \times 4 \times 3 \mathrm{~cm}^{3}$ and appeared nodular on gross examination. Cut section was solid grey yellow, with attached haemorrhagic cyst measuring $1.5 \times 1 \mathrm{~cm}$.

Microscopy of the adnexal mass showed the foci of matted filamentous organisms surrounded by the amorphous eosinophilic material (Figure $2)$. The adjacent tissue showed suppurative inflammation. Section from the cyst showed a haemorrhagic corpus leuteum. Sections from uterus and cervix were unremarkable. A diagnosis of ovarian actinomycosis was made.

Post-operatively the patient was put on IV penicillin for a week, was discharged and was on oral penicillin for 2 weeks. 3 months post-operatively the patient came for a follow up, which was uneventful.

\section{Discussion}

Actinomycosis is a chronic suppurative granulomatous disease caused by any of several anaerobic organisms from the genus Actino-
Figure 3a. $\mathrm{H}$ and $\mathrm{E}$ stain showing the actinomycotic colonies
Figure 3b. Actinomycotic colonies highlighted on Gomori's Methanamine stain

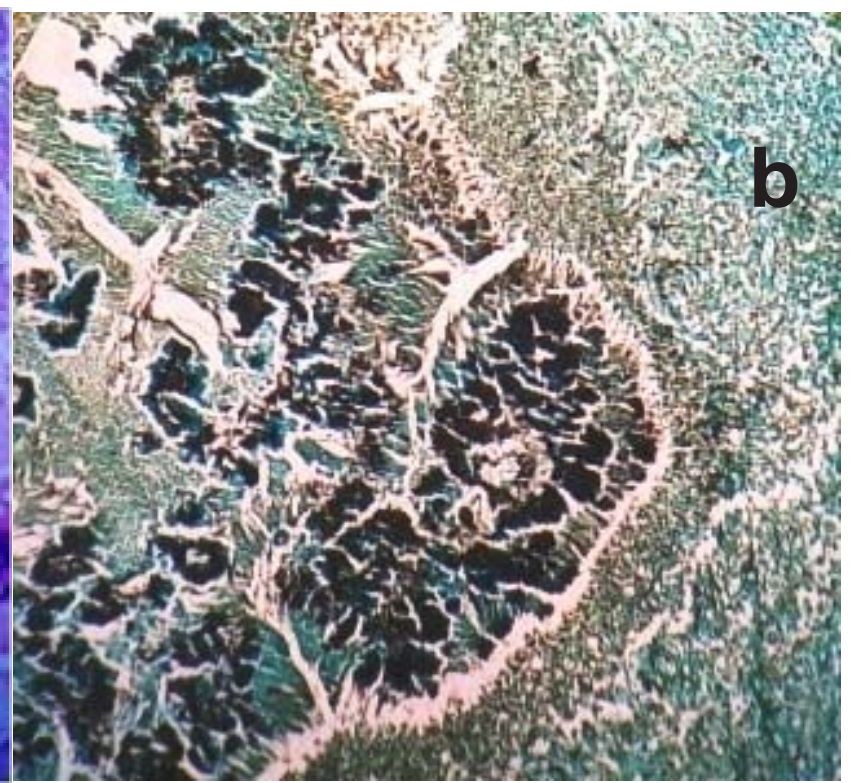

\section{Gomori's Methanamine stain}

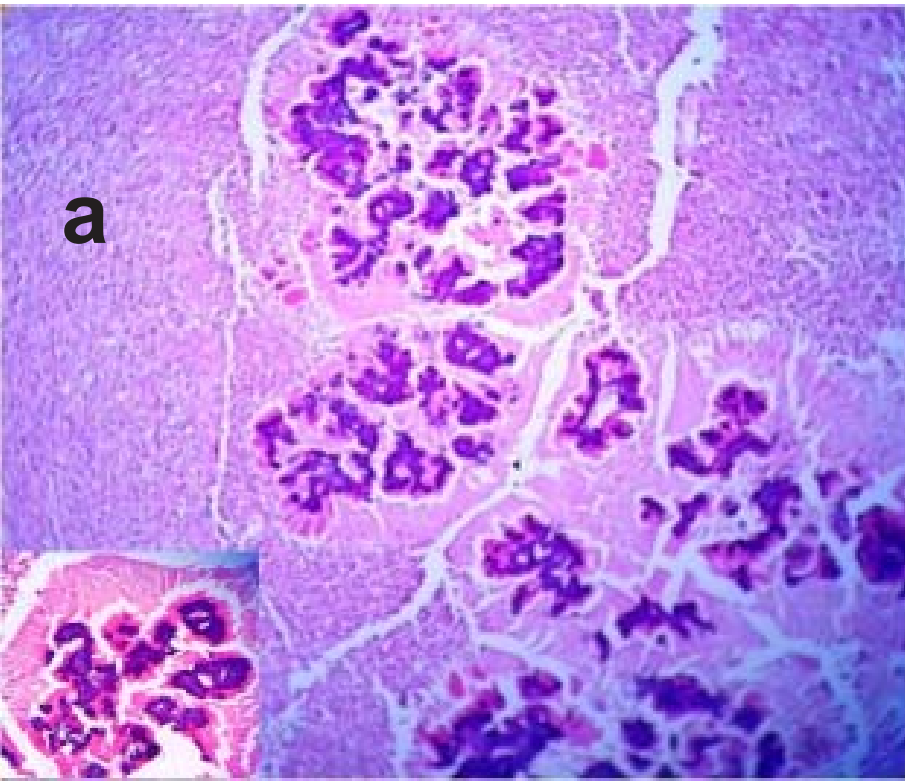


myces. Although previously thought to be a fungal infection, these organisms are gram positive, filamentous bacteria. Disease in humans is caused mainly by Actinomyces Israeli ${ }^{1}$. They are opportunistic pathogens causing infection after disruption of mucous membrane and spreads into surrounding tissue regardless of tissue planes. The most frequent site of human infection is cervicofacial region (40 $50 \%)$, thorax $(15 \%)$, abdomen $(20 \%)$ and pelvis $(3 \%)^{2}$. Pelvic actinomycosis is rare and ovarian actinomycosis is rarer as its structure is resistant to surrounding inflammatory disease. It has been assumed that the bacteria enter the ovary when its surface is broken during ovulation ${ }^{3}$.

Pelvic actinomycosis was seen only in a very small percentage ( 2 in $17,734)$ of non IUD users. Hence, actinomycotic colonisation of the female genital tract was regarded as a rare event in women without an IUD ${ }^{3}$. However, there were previous reported cases of ovarian actinomycosis in the absence of IUD ${ }^{4-7}$. Actinomycosis can follow dental infection ${ }^{1}$; the index case had a history of dental infection and artificial incisor fitting 6 months before presentation. Clinical presentation of actinomycosis could be different and common signs and symptoms are abdominal pain, abdominal mass and loss of weight. Biochemically, it shows leucocytosis and elevation of CA-125 can easily be confounded with ovarian carcinoma. Furthermore, elevated CRP with leucocytosis in actinomycosis can mimic pelvic inflammatory disease (PID).

Imaging findings (ultrasonography and (T) of actyinomycosis include solid adnexal masses with some cystic lesions; usually the appearance is nonspecific and difficult to differentiate from those of malignant ovarian tumours. Small, rim-enhancing lesions in the solid part of the mass are suggestive of actinomycosis. Presence of IUD with above imaging findings would increase the degree of suspicion of pelvic actinomycosis. However, it is important to remember that IUDs are also commonly found in other causes of tubo-ovarian abscesses and also actinomycosis can occur many years after IUD removal ${ }^{9}$. In great majority of the cases, diagnosis of actinomycosis is made by examination of the specimen obtained by surgical exploration and resection. Histopathological features are classic and can be highlighted using special stains like PAS, GMS and Gram's. Detection rate of actinomycosis by culture is very low. Treatment of ovarian actinomycosis is surgical resection of the infected tissue, removal of IUD if any and long term based penicillin therapy.

\section{Conclusion}

Diagnosis of ovarian actinomycosis must always be considered in patients with pelvic mass even in the absence of IUD on presentation or previous history of using the same. Although lab findings can mimic PID and ovarian carcinomas, patients with raised platelet count and more solid lesions on imaging, with bowel wall thickening are strong candidates for actinomycotic infections. Both these features were seen in our case. All clinicians must keep a high index of suspicion of this entity, as early and timely diagnosis can save a woman from excessive surgical intervention.

\section{Acknowledgements}

We thank Dr. Padmashree and her team for providing us such a good specimen for our study.We also thank Dr. Nagaragaiah, Dr. Prakash Jain and the staff off radiology department SIMS AND RC for providing us the images pertaining to the case.

\section{REFERENCES}

1. Simsek A, Perek A, Cakcak El, Durgun VA. Pelvic actinomycosis presenting as a malignant pelvic mass. Journal of Medical Case Reports. 2011; 5: 40.

2. Dasgupta S, Ghosh S, Sengupta SG, Sarkar R. Tubo-ovarian actinomycosis: A case report with brief review of literature. Indian Journal of Medical Sciences, 2010; 64: 329-32.

3. Singh S, Batra A, Dua Sand Duhan A. Ovarian actinomycosis: Presenting as ovarian mass without any history of intrauterine copper device. Journal of Global Infectious Diseases, 2012 Oct - Dec; 4(4): 222-3.

4. Sharma S, Valiathan M, Rao L, Pai MV. Endometrial actinomycosis in post menopausal female in the absence of an intrauterine contraceptive device. Journal Of Clinical Diagnosis and Research. 2012, August; 6(6): 1062-3.

5. Marwah S, Marwah N, Singh I,Gupta A, Jaiswal TS. Ovarian actinomycosis in the absence of an intrauterine contraceptive device: An unusual presentation. Acta Obstet Gynecol Scand 2005; 84: 602-3.

6. Burlando SC, Paz LA, De Feo LG, Benchetrit G, Rimoldi D, Predari SC.Ovarian abscess due to actinomycosis $\mathrm{sp}$. In the absence of an intrauterine contraceptive device. Medicina (B Aires) 2001; 61: 577-80.

7. Munjal K, Nnadedkar S, Subedar V, Jain S.Tubo-ovarian actinomycosis mimicking ovarian malignancy: A report of three cases. Letter to the editor. Indian Journal of Pathology and Microbiology. October December 2010; 53(4): 870-1.

8. Matsuda K, Nakajima $H$, Khan KN, Tanigawa T, Hamaguchi D, Kitajima M, Hiraki K, Moriyama S, Masuzaki H. Pre-operative diagnosis of pelvic actinomycosis by clinical cytology. International Journal of Womens Health. 2012; 4: 527-33.

9. Kim SH, Kim SH, Yang DM, Kim KA. Unusual Causes of Tubo-ovarian Abscess: CT and MR Imaging Findings. Radiographics. 2004 Nov-Dec; 24(6): 1575-89. 